This item was submitted to Loughborough's Research Repository by the author.

Items in Figshare are protected by copyright, with all rights reserved, unless otherwise indicated.

\title{
Modelling of the initial stages of the anisotropic adhesive joint assembly process
}

PLEASE CITE THE PUBLISHED VERSION

PUBLISHER

(c) IEEE

LICENCE

CC BY-NC-ND 4.0

\section{REPOSITORY RECORD}

Mannan, Samjid H., David C. Whalley, Adebayo Oluyinka Ogunjimi, and David J. Williams. 2019. "Modelling of the Initial Stages of the Anisotropic Adhesive Joint Assembly Process". figshare.

https://hdl.handle.net/2134/3985. 
This item was submitted to Loughborough's Institutional Repository (https://dspace.lboro.ac.uk/) by the author and is made available under the following Creative Commons Licence conditions.

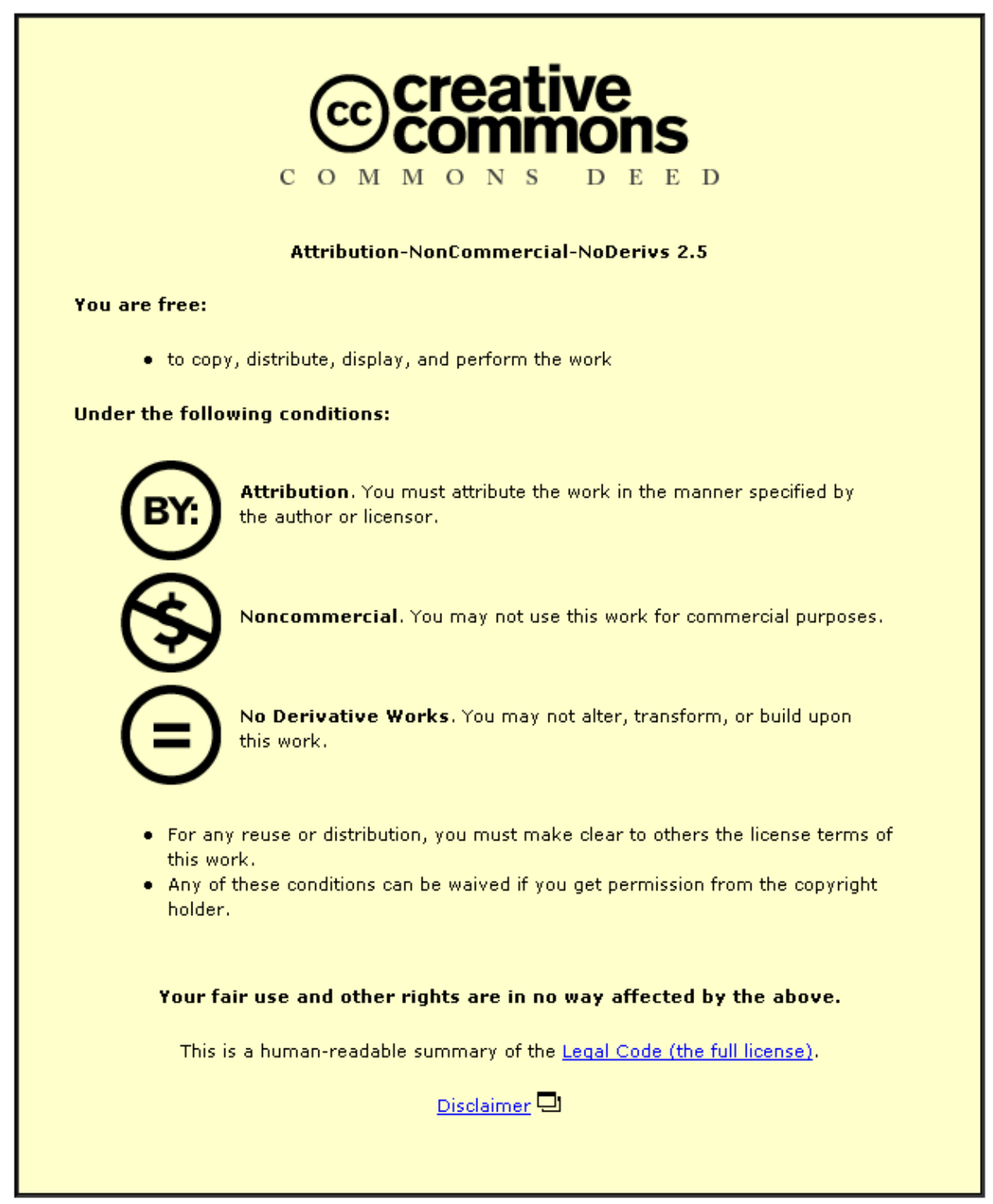

For the full text of this licence, please go to: http://creativecommons.org/licenses/by-nc-nd/2.5/ 


\title{
MC1-3
}

\section{MODELLING OF THE INITIAL STAGES OF THE ANISOTROPIC ADHESIVE JOINT ASSEMBLY PROCESS}

\author{
Samjid H Mannan, David C Whalley, Yinka O Ogunjimi, David J. Williams \\ Department of Manufacturing Engineering, \\ Loughborough University of Technology, \\ Loughborough, LE11 3TU, UK.
}

\begin{abstract}
Industrial experience and academic studies to date indicate the initial stages of the assembly process for anisotropic adhesive joints are particularly significant to the successful formation and long term performance of the completed assembly. The consistency of the process is controlled by the early stages of compression and the resulting distribution of conducting particles is affected by the adhesive resin flow in the early stages of the assembly process. The bump geometry on flip chip assemblies has been found to be critical to the success of the process as it significantly affects the early stages of the adhesive film compression. This paper describes analytical and CFD models which explore the time required to squeeze out the adhesive as a function of applied pressure, temperature ramp rate, and adhesive viscosity. The results of a study on adhesive rheology are presented and used in the flow models. The paper also explores the flow of conducting particles within the adhesive, and hence the final particle distribution underneath the flip chip. This distribution has been shown in the past to significantly affect the probabilities of shorts and opens [1].
\end{abstract}

\section{INTRODUCTION}

Anisotropic Conducting Adhesive (ACA) have been used for some time for connecting LCD drivers to ITO on glass. The advantages offered by this method of connection are high density interconnect, a low temperature process and low cost. More recently ACAs have been used to connect flip-chips to other substrate materials, with varying degrees of success. The main driver towards the use of ACA in this area is the prospect of achieving extremely fine pitch connections at low cost. Added benefits include the elimination of tin/lead solders and elimination of the post assembly cleaning stage.

In ACAs conduction between the pads on the chip, and the lands on the substrate is achieved when pressure and heat are applied to a thin film of adhesive placed between substrate and chip. The pressure causes the adhesive to flow around the pads and lands, and the thickness of the adhesive layer is reduced until contact is made between the pads, the conducting particles in the adhesive and the lands as shown in figure 1 . The excess resin is squeezed out from under the chip.

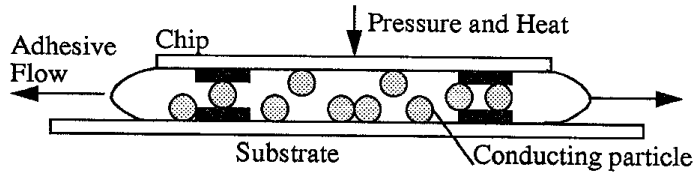

Fig. 1 Conduction mechanism in an ACA.

The adhesive can be either thermoplastic or thermosetting or a combination of the two. By modelling the initial stages of compression, the optimum combination of applied pressure, temperature and time may be found more readily for a given chip geometry, and also the expected distribution of conducting particles on, and around the pads may be predicted. In order to model the adhesive flow, analytical models have been constructed and solved numerically, and Computational Fluid Dynamics (CFD) has also been utilised.

In order to test the analytical model it is first necessary to measure the rheological properties of the ACAs. The properties that were deemed to be most critical were viscosity versus shear rate, viscosity versus temperature and the relaxation time (viscoelastic) of the material. These properties were measured for a typical thermoplastic adhesive and the resulting model predictions were compared against the manufacturer's data sheet. The predictions of the CFD model have also been compared against video evidence of fluid flows and the results have been shown to give good qualitative agreement.

The analytical model of adhesive flow is presented first, followed by the results of the characterisation of one particular ACA. The CFD model is presented next and compared against the video evidence of fluid and particle flow, and finally the conclusions are presented.

\section{ANALYTICAL MODEL OF FLUID FLOW}

The flow of a fluid from between two flat circular disks being pressed together, in the limit that the distance between the plates $(h)$ is much smaller than the radius of the disk $(R)$, has been extensively studied as it is of great interest for lubrication problems. In particular we are interested in the solution for a power law fluid [2] and also the solution for a viscoelastic fluid $[3,4]$. A power law fluid is one in which the shear stress $(\tau)$ depends on the shear rate $(\dot{\gamma})$ in the liquid via a power law, which is a type of behaviour typical of polymer melts:-

$$
\tau=\eta_{0}(\dot{\gamma})^{n}
$$

where $\eta_{0}$ is termed the consistency and $n$ the power law index.

For a Newtonian fluid $n=1$ and $\eta_{0}$ becomes the viscosity of the fluid $(\eta) ; \eta$ is defined as follows:-

$$
\eta=\frac{\tau}{\dot{\gamma}}=\eta_{0}(\dot{\gamma})^{n-1}
$$

Given a fluid which is sufficiently viscous to neglect inertial effects, the equation that predicts the motion of the plates as a function of time $(t)$, in the lubrication limit is known as the Scott equation [2]:-

$$
\frac{d h}{d t}=-\frac{n}{2 n+1}\left(\frac{(n+3) F h^{2 n+1}}{2 \pi \eta_{0} R^{n+3}}\right)^{1 / n}
$$


where $F$ is the force acting on the top disk (bottom plate held rigid), and the other parameters have been defined. This equation can be solved to find the time required to reduce the film thickness from its original value, $h_{0}$, to $h$ :-

$$
t=\frac{2 n+1}{n+1}\left(\frac{2 \pi \eta_{0} R^{n+3}}{F(n+3) h_{0}^{n+1}}\right)^{1 / n}\left(\left(\frac{h_{0}}{h}\right)^{\frac{n+1}{n}}-1\right)
$$

Equation 4 can be used to investigate how various factors affect the time required to squeeze out the adhesive if we consider the bottom plate to be the substrate and the top plate to be the chip. One notable fact is that for a constant value of applied pressure $\left(F / R^{2}\right)$, the radius of the chip has a strong effect on the squeezing time $-t \propto R^{(1+n) / n}$. The Scott equation can also be used to predict the pressure distribution on the substrate and chip [6].

Chips are generally square or rectangular rather than circular and this of course will effect the squeezing time. Likewise the effect of the substrate lands (and also bumps on the flip chip if it is bumped) will not in general be negligible. However, modelling the flow around the lands (and bumps) requires CFD techniques. These issues will be discussed later.

In deriving eq. 4 it has been assumed that the temperature of the adhesive is held fixed while the pressure is applied, as is usual for thermoplastics. If this is not the case and the temperature is being ramped up as is usual for thermosets, then we need to take into account the dependence of viscosity on temperature. A reasonable approximation is that, above the glass transition temperature, the viscosity depends exponentially on temperature:-

$$
\eta=\eta_{0} e^{-b\left(T-T_{1}\right)}(\dot{\gamma})^{n-1}
$$

where $b$ is a constant, $T$ is the temperature and $T_{1}$ is the reference temperature at which eq. 1 is valid. We will further assume that the temperature is ramped up linearly with time:-

$$
T=T_{1}+k t
$$

We can now substitute eq. 6 and eq. 5 into eq. 3 - the consequence being that we must replace $\eta_{0}$ by $\eta_{0} e^{-b k t}$ in eq. 3 . The solution of eq. 3 now becomes:-

$$
t=\frac{n}{b k} \ln \left(1+\frac{b k}{n} \xi\right)
$$

where $\xi$ is given as:-

$$
\xi=\frac{2 n+1}{n+1}\left(\frac{2 \pi \eta_{0} R^{n+3}}{F(n+3) h_{0}^{n+1}}\right)^{1 / n}\left(\left(\frac{h_{0}}{h}\right)^{\frac{n+1}{n}}-1\right)
$$

which is just the right hand side of eq.4 again. In the limit that $k \rightarrow 0$, which is the limit that temperature is held constant, we see that eq. 6 reduces to eq. 4 as expected. However if the term $b k \xi / n$ in eq. 7 is large compared to 1 , then the most important processing parameter is $k$ and hence the rate at which the adhesive is squeezed out is determined primarily by the temperature ramp rate as $1 / k$.

Thus far we have assumed that the viscoelastic nature of the adhesive can be ignored. This is a reasonable assumption if the relaxation time of the fluid $(\lambda)$ is small compared to the time the squeezing takes to be completed [3]. If this is not the case, then the effect of viscoelasticity is to increase the processing time beyond that predicted by the Scott equation. A detailed model of the fluid motion in this case can be found in [4]. The
ACA which we have characterised has however been found to have a relaxation time smaller than its processing time as detailed in the next section.

\section{RHEOLOGICAL CHARACTERISATION}

The rheological characteristics of the adhesive needed for modelling the flow of adhesive under the chip are the variation of viscosity with shear rate (which gives us $\eta_{0}$ and $n$ in eq.1), the variation of viscosity with temperature (which gives us $b$ in eq.5) and the relaxation time of the material, $\lambda$.

The material chosen for characterisation was a thermoplastic containing $10-15 \%$ by volume of 10 micron spherical conducting particles. This material is in commercial production and use. A thermoplastic adhesive was chosen because the same sample may be repeatedly tested, while for a thermoset, all experiments would have to be designed to allow for the change in rheological properties as the material cures.

A Bohlin controlled stress rheometer was used for the rheological measurements. Parallel plate geometry was used and the measurements taken included both stress viscometry (where the bottom plate was fixed and the top plate rotated by applying specified torque) and oscillation experiments for the characterisation of linear viscoelasticity (where the top plate underwent small amplitude angular oscillations at a specified frequency). The sample thickness was 70 microns - a rule of thumb in stress viscometry is that the sample thickness should be at least 10 times the suspended particle diameter, but because the ratio is 7 in our case, the experiments were repeated with adhesive without the particles to provide a consistency check.

A typical polymer melt [5] has a power law index, $n$ of about 0.6 . However the results of the stress viscometry experiments showed that the ACA was almost Newtonian - $n$ was found to be 0.9 . $\eta_{0}$ was found to be $4.9 \times 10^{4} \mathrm{~Pa} . \mathrm{s}^{n}$ which is of a similar order of magnitude to typical polymer melts. The high value of $n$ is probably not coincidence - during flow, the highest shear rates are found around lands and bumps, so that if the material had a low value of $n$, the adhesive could not produce a strong viscous drag to keep the particles on the pads. Thus one desirable characteristic of an ACA is a high power law index. The variation of viscosity with shear rate is shown in fig. 2 for both the ACA and the unfilled polymer.

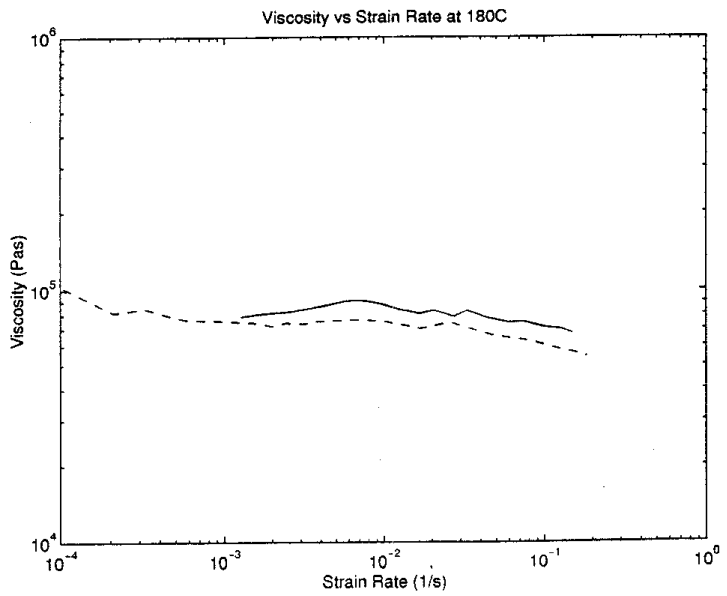

Fig.2 Viscosity for ACA (line) and polymer (dash). 
The similarity between the two curves suggests that the addition of the conducting particles does not alter the rheology of the adhesive very significantly in specimens of this thickness - the increase in viscosity of approximately $28 \%$ is as expected from a suspension filled with spheres to a volume fraction of $10-15 \%$ (an increase of around 2.5 times the volume fraction is typical [7]). The effect of the particles on rheology is expected to be more pronounced for thinner films. Fig.2 also shows that the fluid is approximately Newtonian up to a strain rate of $0.01 \mathrm{~s}^{-1}$ (strain rates over $10 \mathrm{~s}^{-1}$ are expected during processing).

The variation of viscosity with temperature is shown in figure 3. The gradient of this graph is given by $-b / n$ (see eq.5) because the measurements are taken at constant stress and not constant strain rate. We thus find a value of $b$ of 0.058 . We may now rewrite eq. 5 with $180^{\circ} \mathrm{C}$ as our reference temperature:-

$$
\eta=4.9 \times 10^{4} e^{-0.058(T-180)}(\dot{y})^{-0.1}
$$

This equation defines the rheological characteristics of the ACA provided that the relaxation time of the material is much smaller than the process time.

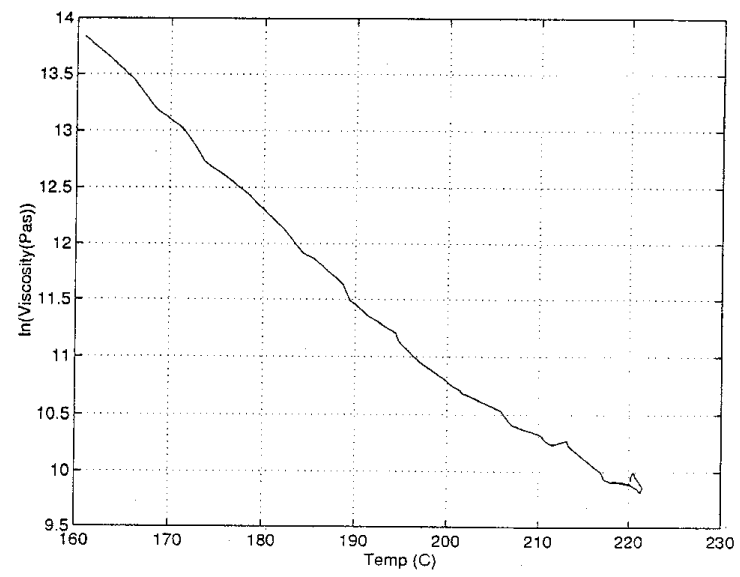

Fig.3 Viscosity vs Temperature for ACA

Figure 4 shows the variation of storage modulus (G') and loss modulus (G”) (see eg. [5]) with frequency at $180^{\circ} \mathrm{C}$. An estimate of the relaxation time of the material can be made by finding the frequency $(f)$ at which these two are equal and from figure 4 we see that they are close at $0.3 \mathrm{~Hz}$; the relaxation time is equal to $1 /(2 \pi f)$ which is approximately $0.5 \mathrm{~s}$. This is much smaller than the process time of the material at $180^{\circ} \mathrm{C}$ but at $220^{\circ} \mathrm{C}$ the manufacturers data sheet suggests processing times of order $1 \mathrm{~s}$ - however the relaxation time also decreases as the temperature is increased and at $200^{\circ} \mathrm{C}$ the relaxation time is approximately $0.15 \mathrm{~s}$ so that viscoelasticity does not place severe restrictions on the processing time.

The data contained in eq. 9 can now be used to predict the processing time for a given pressure and temperature. The original film thickness is 50 microns and it is reasonable to assume a final film thickness of 35 microns. If we take a chip of radius $0.5 \mathrm{~cm}$ and a loading force of $200 \mathrm{~N}$, then we find from eq. $4 \mathrm{a}$ processing time of $163 \mathrm{~s}$. At $200^{\circ} \mathrm{C}$ this reduces to $45 \mathrm{~s}$, while at $220^{\circ} \mathrm{C}$ the process time is $13 \mathrm{~s}$. At $175^{\circ} \mathrm{C}$ with a chip of radius of
$0.127 \mathrm{~cm}$ and a loading force of $9.6 \mathrm{~N}$ the processing time is predicted to be $17 \mathrm{~s}$ compared to the manufacturer's recommendation of 10 s. The discrepancy is probably due to not knowing the exact geometry used for producing the manufacturer's datasheet.

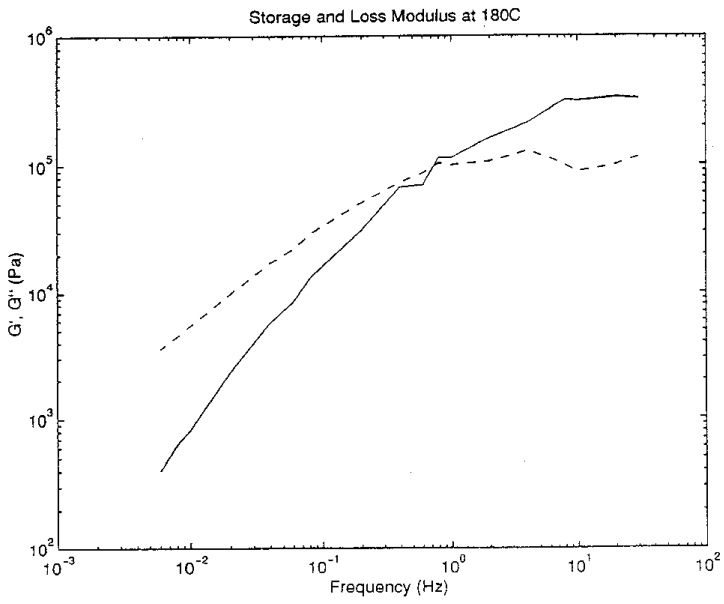

Fig.4 Storage (line) and loss (dash) modulus for ACA

\section{CFD and FLOW VISUALIZATION}

The previous sections have been concerned mainly with predicting the time required to squeeze out the adhesive from beneath the chip, and has generally ignored the presence of the conducting particles in the adhesive. The distribution of these particles around the pads and lands after the initial stages of compression are complete is however vital as this distribution determines the conducting properties of the joint. An attempt has been made to predict the overall change in volume fraction before and after compression using analytical methods [8]. This work showed that the volume fraction, $\phi$, is expected to be, after compression, in the range $1.03 \phi$ to $0.7 \phi$ depending on the exact material properties and geometry.

The detailed distribution of particles around the pads must however be found using CFD and flow visualization methods. CFD can also be used to analyse the assumptions used in the analytical modelling. Using FIDAP, a commercially available CFD package, the validity of the Scott equation has been checked as a function of $R / h$ and it has been found that when $R / h=10$ the errors are of order $1 \%$, when $R / h=5$ the errors are of order $2 \%$ and when $R / h=2.5$ the errors have grown to $10 \%$ (with $n$ close to 1 ). With typical flip-chip geometries the lowest value of $R / h$ is likely to be around 25 so that there is no problem with the Scott equation, but for individual bump geometries the condition $R / h>5$ is unlikely to be achieved.

Another assumption made in the analytical models is that the chip can be modelled as circular rather than square. Using CFD it is possible to check this assumption and we find that the flow patterns on the corners of the chip are significantly different from those at the midpoint of the edges. Figure 5 illustrates the flow patterns found on a $4.5 \mathrm{~mm}$ square unbumped chip with 24 pads (pitch $0.5 \mathrm{~mm}$, height 30 microns) when the distance between chip and substrate is 40 microns. Figure 6 shows the effect of removing the corner pads (the half pad shown on the left of fig.5) and we see that this does not result in a more even distribution of fluid flows. 
The lands themselves provide a pressure locking effect; for a given approach velocity of the chip onto the substrate the effects of the lands is to increase the required applied pressure by $36 \%$.

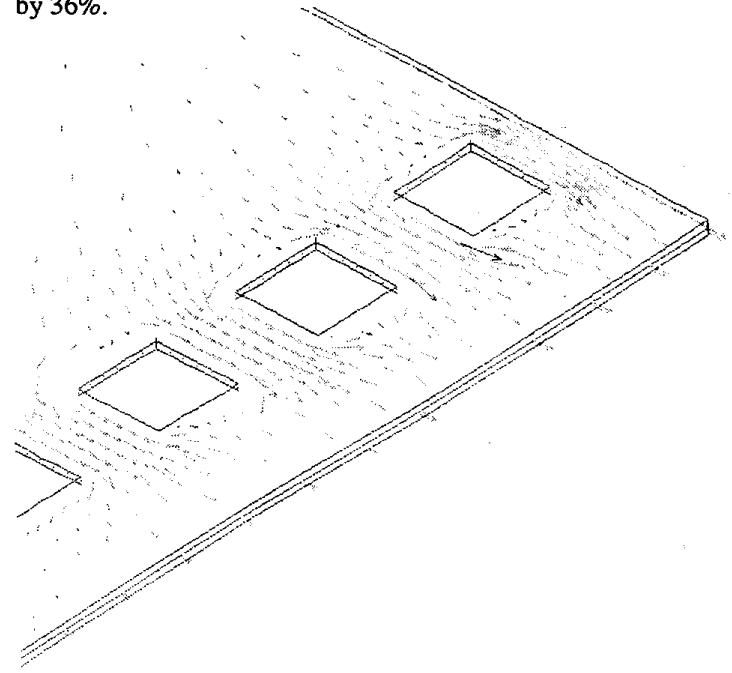

Fig.5 CFD Prediction of velocities field around lands.

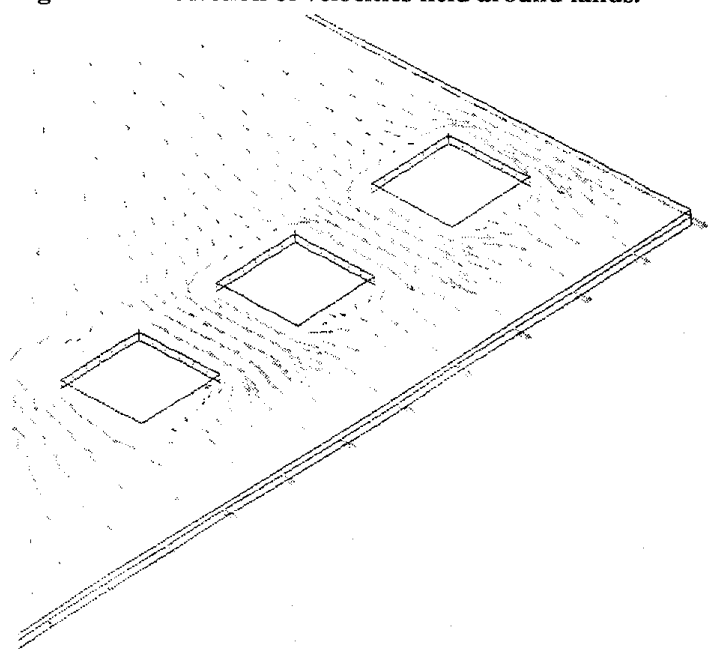

Fig.6 CFD Prediction for velocity field without corner land.

Flow visualization experiments can be used to check the accuracy of the CFD model. To observe the flow patterns directly, a glass substrate was used to simulate an unbumped chip, and a camera placed to record the movement of the adhesive between the glass and the substrate (FR4 with 30micron high copper lands). The CFD model predicts that particles should only be squeezed out from under the pads in the final stages of compression and this is observed experimentally. Furthermore particles leaving the pads from the edge nearest the edge of the chip are predicted to have a speed 4.5 times greater than those leaving the pad from the side nearest the centre of the chip; 4.0 is the ratio observed, in agreement with the prediction.

The asymmetry in the velocity field on the lands suggests that there will be fewer particles on the half of the land towards the chip edge than there are towards the chip centre. Experimentally the difference appears to be very small. On a 250 micron square pad around 130 particles were found; 2000 particles per $\mathrm{mm}^{2}$.

\section{CONCLUSIONS}

An analytical model has been built which shows how the squeezing time is affected by various process parameters and geometry. The results highlight that the chip size may have a large effect on process time. This shows that typical manufacturing process data is insufficient to determine the correct process conditions for a specific geometry. The model predictions have been shown to give reasonable agreement with experiment in one case where the adhesive rheology has been characterised and viscoelastic effects have been shown to be unimportant. It has been assumed throughout that the adhesive film height is the distance between the chip and substrate (i.e. not the distance between pads and lands); this is discussed more fully elsewhere [8].

A CFD model has been constructed which agrees with flow visualization experiments, and which has been used to see the effect of changing the land layout on flow patterns. The model correctly predicts the flow patterns on the lands.

\section{ACKNOWLEDGEMENTS}

The authors acknowledge the support of the Engineering and Physical Sciences Research Council (EPSRC) and a consortium of industrial partners; Cooksons Technology Centre and their associated company Alpha Metals Advanced Products, Eltek Semiconductors Ltd., Lucas Electronics, Rolls-Royce Plc, Design 2 Distribution Ltd., IBM (UK) Ltd. and Mitel Telecom. Special thanks go to Gary Nichols of Cooksons Technology who arranged the use of Cooksons" rheological equipment, and Johan Liu of the IVF, Sweden who gave impetus to our flow visualization experiments by getting there first!

\section{REFERENCES}

[1] D.J. Williams, D.C. Whalley, O.A. Boyle, A.O. Ogunjumi, "Anisotropic Conducting Adhesives for Electronic Interconnection" Soldering and surface mount technology, no. 14, pp.4-8, June 1993.

[2] J.R. Scott, Trans. Inst. Rubber Ind., vol. 8, pp. 481-493, 1932 .

[3] P. J. Leider, "Squeezing Flow Between Parallel Disks. II.", Ind. Eng. Chem. Fundam., vol. 13, pp. 342-346, 1974.

[4] M.A. McMclelland and B.A. Finlayson, "Squeezing Flow of Highly Viscous Polymers", Journal of Rheology, vol. 32, pp. 101-133, 1988.

[5] H.A. Barnes, J.F. Hutton, K. Walters, An Introduction to Rheology, Elsevier, 1989, ch. 2, p22.

[6] A.O. Ogunjimi, S. H. Mannan, D.C. Whalley, D.J Williams, "The Assembly Process for Anisotropic Conductive Joints Some New Experimental and Theoretical Results" in Proceedings of Latest Achievements in Conducting Adhesive Joining in Electronics Packaging, 1995, pp. 129-134.

[7] A. Einstein, "Eine neue Bestimmung der Molekuldimension," Ann. Physik, vol 19, pp. 289-306, 1906.

[8] S. H. Mannan, A.O. Ogunjimi, D.C. Whalley, D.J Williams, "Modelling Resin Flow in Flip Chip Assembly with Anisotropic Conducting Adhesive," Loughborough University Processes Group Technical Report 95/5, 1995. 